\title{
Combined MW-IR Precipitation Evolving Technique (PET) of convective rain fields
}

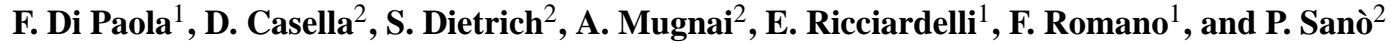 \\ ${ }^{1}$ Istituto di Metodologie per l'Analisi Ambientale, CNR, Rome, Italy \\ ${ }^{2}$ Istituto di Scienze dell' Atmosfera e del Clima, CNR, Rome, Italy \\ Correspondence to: F. Di Paola (francesco.dipaola@imaa.cnr.it)
}

Received: 16 July 2012 - Revised: 10 October 2012 - Accepted: 12 October 2012 - Published: 29 November 2012

\begin{abstract}
This paper describes a new multi-sensor approach for convective rain cell continuous monitoring based on rainfall derived from Passive Microwave (PM) remote sensing from the Low Earth Orbit (LEO) satellite coupled with Infrared (IR) remote sensing Brightness Temperature (TB) from the Geosynchronous (GEO) orbit satellite. The proposed technique, which we call Precipitation Evolving Technique (PET), propagates forward in time and space the last available rain-rate (RR) maps derived from Advanced Microwave Sounding Units (AMSU) and Microwave Humidity Sounder (MHS) observations by using IR TB maps of water vapor $(6.2 \mu \mathrm{m})$ and thermal-IR $(10.8 \mu \mathrm{m})$ channels from a Spinning Enhanced Visible and Infrared Imager (SEVIRI) radiometer. PET is based on two different modules, the first for morphing and tracking rain cells and the second for dynamic calibration IR-RR. The Morphing module uses two consecutive IR data to identify the motion vector to be applied to the rain field so as to propagate it in time and space, whilst the Calibration module computes the dynamic relationship between IR and RR in order to take into account genesis, extinction or size variation of rain cells. Finally, a combination of the Morphing and Calibration output provides a rainfall map at IR space and time scale, and the whole procedure is reiterated by using the last RR map output until a new MW-based rainfall is available. The PET results have been analyzed with respect to two different PM-RR retrieval algorithms for seven case studies referring to different rainfall convective events. The qualitative, dichotomous and continuous assessments show an overall ability of this technique to propagate rain field at least for $2-3 \mathrm{~h}$ propagation time.
\end{abstract}

\section{Introduction}

The ability to estimate convective precipitation with a high level of accuracy, by using Passive Microwave (PM) from radiometers onboard satellites, has been well known for several years (Ebert et al., 1996; Smith et al., 1998; Kummerow et al., 2001), since the launch of the first Special Sensor Microwave/Imager (SSM/I) radiometer in 1987 - and especially after the launch of the Tropical Rainfall Measuring Mission (TRMM) space observatory in 1997. However, due to diffraction, in order to achieve spatial resolutions consistent with the horizontal variability of the geophysical parameters retrievable from $\mathrm{PM}$, passive millimeter radiometers have been flown, so far, on Low Earth Orbiting (LEO) satellites. This brings about coarse temporal sampling that ranges from more than 10 remote sensing per day at high latitudes to 1-2 per day at low latitudes, for any given location over the Earth. Obviously, this temporal sampling does not provide satisfactory coverage of observations for some geophysical parameters rapidly evolving such as convective precipitation.

To solve this problem, some national and international space agencies have made preliminary attempts to evaluate the scientific/technological prospect of sub-hourly rainfall observations from space by using millimeter-submillimeter MW radiometers onboard geostationary (GEO) satellites (e.g. Bizzarri et al., 2007; Di Paola and Dietrich, 2008), even if the launch of such satellites has not be planned for the present.

To attenuate the problem arising from the coarse temporal sampling of microwave remote sensing, several MW radiometers are presently operational on LEO satellites, as shown in Table 1. Despite this large constellation of satellites embarking MW radiometers, the real-time precipitation 
Table 1. List of the main LEO satellites carrying MW radiometers.

\begin{tabular}{lllrll}
\hline Satellite & Operator & LTAN & Mean Altitude & Launch date & MW instrument payload \\
\hline GCOM-W1 & JAXA & $13: 30$ & $700 \mathrm{~km}$ & 18 May 2012 & AMSR-2 \\
Suomi-NPP & USA/NOAA/NASA & $13: 30$ & $833 \mathrm{~km}$ & 27 Oct 2011 & ATMS \\
Megha-Tropiques & ISRO + CNES & non-sun-synch. & $865 \mathrm{~km}$ & 12 Oct 2011 & MADRAS/SAPHIR \\
& & $\left(20^{\circ}\right.$ inclination $)$ & & & \\
FY-3B & CHINA/CMA & $13: 38$ & $836 \mathrm{~km}$ & 5 Nov 2010 & MWRI/MWHS \\
DMSP-F18 & USA/NOAA-DOD & $20: 08$ & $850 \mathrm{~km}$ & 18 Oct 2009 & SSMIS \\
NOAA-19 & USA/NOAA & $13: 32$ & $870 \mathrm{~km}$ & 6 Feb 2009 & AMSU/MHS \\
FY-3A & CHINA/CMA & $20: 22$ & $836 \mathrm{~km}$ & 27 May 2008 & MWRI/MWHS \\
Metop-A & EUMETSAT & $21: 30$ & $837 \mathrm{~km}$ & 19 Oct 2006 & AMSU/MHS \\
DMSP-F17 & USA/NOAA-DOD & $17: 37$ & $850 \mathrm{~km}$ & 4 Nov 2006 & SSMIS \\
NOAA-18 & USA/NOAA & $14: 22$ & $854 \mathrm{~km}$ & 20 May 2005 & AMSU/MHS \\
DMSP-F16 & USA/NOAA-DOD & $18: 51$ & $850 \mathrm{~km}$ & 18 Oct 2003 & SSMIS \\
NOAA-17 & USA/NOAA & $20: 43$ & $810 \mathrm{~km}$ & 24 Jun 2002 & AMSU \\
Aqua & NASA & $13: 30$ & $705 \mathrm{~km}$ & 4 May 2002 & AMSR-E/AMSU-A/HSB \\
NOAA-16 & USA/NOAA & $19: 45$ & $849 \mathrm{~km}$ & 21 Sep 2000 & AMSU \\
DMSP-F15 & USA/NOAA-DOD & $17: 06$ & $850 \mathrm{~km}$ & 12 Dec 1999 & SSM/I \\
TRMM & NASA/JAXA & non-sun-synch. & $402 \mathrm{~km}$ & 28 Nov 1997 & TMI \\
& & $\left(35^{\circ}\right.$ inclination) & & & \\
DMSP-F14 & USA/NOAA-DOD & $15: 47$ & $852 \mathrm{~km}$ & 4 Apr 1997 & SSM/I \\
\hline
\end{tabular}

monitoring obtained by using only a passive MW onboard satellite, is still not possible, due to the rapid variability of rain field with respect to the temporal frequency of LEO satellite overpass.

In the last twenty years, various algorithms have been developed by combining frequent IR observations from GEO satellites with accurate MW-based precipitation estimates (Adler et al., 1993; Xu et al., 1999; Bellerby et al., 2000; Sorooshian et al., 2000; Huffman et al., 2001; Miller et al., 2001; Kuligowski, 2002; Turk and Miller, 2005). While some MW-IR combined techniques focus on rain-rate estimates on the GEO time scale (every $15 / 30 \mathrm{~min}$ ) - e.g. the Naval Research Laboratory (NRL) blended algorithm (Turk et al., 2002; Turk and Mehta, 2007; Torricella et al., 2007), the University of Birmingham algorithm (Kidd et al., 2003, 2007), and the Climate Prediction Center (CPC) morphing algorithm (CMORPH - see Joyce et al., 2004, 2007; see also Kubota et al., 2007), other techniques aim at 3-h or longer time scales precipitation estimates (e.g. Adler et al., 1994; Huffman et al., 2007).

In this paper, we describe the PET algorithm which is a new combined MW-IR technique that employs IR observations from GEO satellite to propagate forward in time the rain field of convective storms. This technique uses the latest available rain-rate estimates provided by MW observations from space and produces rain-rate maps at the GEO time and space resolution for real-time purposes. PET can be considered as a combination and evolution of the CMORPH technique and NRL blended technique using two separate modules which we call Morphing and Calibration. Even though both the PET Morphing module and CMORPH propagate rain field by motion vectors derived from GEO IR data, the basic innovation of the Morphing described in this paper with respect to CMORPH is that the former requires only one MW-estimated rain field to allow a quasi real-time monitoring, while the latter needs two MW-estimated rain fields to generate the in-between rain fields, and obviously it does not allow real-time applications.

The basic innovation of the PET Calibration module with respect to NRL blended technique is that it is based on the Global Convective Diagnostic (GCD) approach (Mosher, 2001,2002 ) rather than on a single thermal infrared channel. GCD is a method for recognizing deep convection from geostationary satellite images, both day and night, based on the difference from thermal infrared and water vapor channels. By using Precipitation Radar (PR) onboard the Tropical Rainfall Measurement Mission (TRMM), Martin et al. (2008) noted that GCD generally produces more accurate deep-convection observation than any other monospectral infrared convective scheme.

As shown in a previous work (Casella et al., 2012) with the PM-GCD technique, the combined use of SEVIRI $6.2 \mu \mathrm{m}$ 
and $10.8 \mu \mathrm{m}$ brightness temperatures allows to recognize convective areas in a better way than the use of a single IR channel, highly reducing the number of false alarms such as those caused by the cirrus or others non-precipitating clouds that are one of the error main sources of some IR-based rainrate estimate techniques. Another reason to use a $6.2 \mu \mathrm{m}$ and $10.8 \mu \mathrm{m}$ brightness temperature combination instead of a single thermal-IR radiance is that the latter shows very small variation, while GCD value tends to decrease from the core convective cell to the anvil region. With respect to PM-GCD that uses a statistical relationship GCD-RR, PET uses both a deterministic and statistical approach by means of Morphing and Calibration modules, respectively, to propagate the rain field. In the first steps, as better explained in Sect. 4, PET uses mainly the Morphing module. The Calibration module becomes relevant only after some propagation steps. As a matter of fact, at least at the beginning of propagation, the PET output is more consistent with the MW-based rain field when compared with PM-GCD.

It is worth considering that PET is not a method for estimating rain rates, rather a technique that creates spatially and temporally complete rain field information by using existing precipitation products, like those derived from MW observations. In this paper we use MW-derived rain rates from the Advanced Microwave Sounding Unit-A and -B (AMSU-A, AMSU-B) and Microwave Humidity Sounder (MHS) radiometers onboard NOAA and MetOp operational LEO satellites, in conjunction with IR brightness temperature from the Spinning Enhanced Visible and Infrared Imager (SEVIRI) radiometer onboard the geostationary METEOSAT Second Generation (MSG) satellite.

Section 2 briefly reviews AMSU/MHS and SEVIRI data, the two MW-based precipitation estimation algorithms used in this study and the GCD technique used to identify convective areas. Section 3 introduces a heavy rainfall occurred over Sicily in 2009 used as a case study to better explain the algorithm and to show its preliminary results. Sections 4 and 5 describe how PET algorithm works, analyzing both Morphing and Calibration modules and its application to the selected case study. In Sect. 6 a preliminary assessment of PET is carried out by comparing the PET rain-rate maps with the corresponding AMSU/MHS-based retrievals with reference to seven convective storms occurred in the Mediterranean region between 2009 and 2012. Section 7 summarizes these studies and draws the conclusions.

\section{Data and algorithm}

Since our purpose is to develop a method for real-time rain rates monitoring for convective events, we have selected only the MW radiometers onboard operational satellites - the cross-track scanning AMSU/MHS sensors onboard NOAA18, -19 and MetOp-A sun-synchronous near-polar-orbiting operational satellites, coupled with IR data from SEVIRI onboard MSG. Even if there are others satellites equipped with MW sensors, for this study only NOAA and MetOp satellites have been used because the other satellites have some radiometer malfunctions (see http://www.osdpd.noaa. gov and http://www.oso.noaa.gov) or high date latency making them unsuitable for real-time applications. However, the use of the latest sensors will be included in PET as soon as the proper retrieval algorithms is developed.

A detailed description of AMSU/MHS radiometers can be found in Kramer (2002) and in Kleespies and Watts (2007). Here we wish to give some essential information that the reader may find useful. AMSUB/MHS are cross-track radiometers, providing images with constant angular sampling across tracks, which implies that the Instantaneous Field Of View (IFOV) elongates as the beam moves from nadir toward the edge of the scan. For all frequencies, AMSU-A IFOV is approximately $(48 \times 48) \mathrm{km}^{2}$ at nadir and increases up to $(80 \times 150) \mathrm{km}^{2}$ at the edge of the $2250 \mathrm{~km}$ swath, whilst AMSU-B and MHS IFOV are approximately $(16 \times 16) \mathrm{km}^{2}$ at nadir and increase up $(27 \times 50) \mathrm{km}^{2}$ at the edge of scan line. AMSU-A was originally developed for atmospheric temperature sounding and most frequencies lie in the $\mathrm{O}_{2}$ absorption band at $54 \mathrm{GHz}$. AMSU-B and MHS were developed for humidity sounding and most frequencies lie in the $\mathrm{H}_{2} \mathrm{O}$ absorption band at $183 \mathrm{GHz}$. In the presence of precipitating clouds, temperature and humidity profiling mission fails and precipitation is observed instead.

In this paper we adopt two AMSU/MHS precipitation retrieval algorithms, in order to highlight the different results from PET algorithm for the same case studies but different rain-rate estimates. The first algorithm was developed at the Massachusetts Institute of Technology (MIT) (Surussavadee and Staelin, 2008a). This algorithm is based on neural networks trained with simulated $T_{B}$ at AMSU/MHS frequencies for 122 representative storms covering a wide range of precipitation types, between July 2002 and June 2003. Simulated $T_{B}$ are obtained by using a two-stream Mie-scattering Radiative Transfer Model (RTM) (Rosenkranz, 2002), applied to meteorological fields simulated with a mesoscale Numerical Weather Prediction (NWP) model (MM5) initialized with the National Centers for Environmental Prediction (NCEP) data. This algorithm uses a Principal Component Analysis of $T_{B}$ and the outputs produced are rain-rate estimates at AMSU-B/MHS grid.

The algorithm was originally validated through numerical comparisons with precipitation products derived from AMSR-E onboard the Aqua satellite, SSM/I onboard the DMSP F-13, F-14 and F-15 satellites, TMI onboard the TRMM satellite and surface precipitation rates product (NOWRAD) by using the Next-Generation Weather Radar (NEXRAD) programme (Surussavadee and Staelin, 2008b). The second algorithm was developed at the Institute of Methodologies for Environmental Analysis of the National Research Council of Italy (IMAA-CNR) (Di Tomaso et al., 2009). It is entirely based on AMSU-B/MHS observations 
and it does not require the integration of additional sensors wich could deteriorate the spatial resolution. It uses both the radiometric observations made at window channel (89 and $150 \mathrm{GHz}$ ) and those made in the water vapor band $(183 \mathrm{GHz})$. This algorithm has been validated on a large number of case studies at middle and low latitudes as well as over land and land/water surfaces. Precipitation estimates exhibit a very good agreement with ground-based observations in the detection of rainfall. The probability of detection of precipitation is $75 \%$ and $90 \%$ for rain rates greater than $1 \mathrm{~mm} \mathrm{~h}^{-1}$ and $5 \mathrm{~mm} \mathrm{~h}^{-1}$, respectively.

SEVIRI is the main payload onboard MSG geostationary satellite, launched by Eumetsat since 2003 above the Guinea Gulf. This radiometer consists of two visible channels centered at 0.6 and $0.8 \mu \mathrm{m}$, one near infrared channel centered at $1.6 \mu \mathrm{m}$, eight infrared channels centered at 3.9, 6.2, 7.3, 8.7, $9.7,10.8,12.0$ and $13.4 \mu \mathrm{m}$ and finally one visible broadband channel at $0.5 / 0.9 \mu \mathrm{m}$ called the High Resolution Visible channel (HRV). SEVIRI combines a very high temporal resolution of 15 min over the whole Earth disk with a good spatial sampling of $(1 \times 1) \mathrm{km}^{2}$ for HRV and $(3 \times 3) \mathrm{km}^{2}$ for the others channels at sub-satellite point. The level 1.5 data used for this paper have been corrected from radiometric and geometric non-linearity, geolocated and calibrated (Muller, 2007). A detailed description of SEVIRI radiometers can be found in Aminou et al. (1997). The GCD technique used for PET algorithm is based on the TB difference between thermal-infrared (IR, $10.8 \mu \mathrm{m}$ ) and water vapor (WV, $6.2 \mu \mathrm{m}$ ) channels (i.e., $\Delta \mathrm{TB}^{\mathrm{GCD}}=\mathrm{TB}^{\mathrm{IR}}-\mathrm{TB}^{\mathrm{WV}}$ ) and was developed to identify deep-convection areas from GEO observations. Even if this method originally assigns deep convection when the difference $\triangle \mathrm{TB}^{\mathrm{GCD}}$ is lower than a given threshold (usually, $1 \mathrm{~K}$ ), in this paper we use a dynamic relationship between the whole $\triangle \mathrm{TB}^{\mathrm{GCD}}$ and rain-rate maps to identify and propagate the convection areas for both Morphing and Calibration modules.

\section{A case study}

To better explain this method, the PET algorithm is described in the following section with reference to a severe convection storm that originated on 30 September 2009 with a depression developed over Northern Africa and a consequent warm advection from the Sahara to the Mediterranean region, culminating in an extraordinary intense rainfall on 1 October 2009 in Sicily in southern Italy. The heavy rainfall covered approximately the region $(35.0-42.5)^{\circ} \mathrm{N}$ latitude and $(7.5-17.5)^{\circ} \mathrm{E}$ longitude and discharged an amount of more than $200 \mathrm{~mm}$ of rain in southern Italy, causing the death of 35 people due to a large landslide affected the area around Messina - see Dietrich et al. (2011) for a detailed description of this case study.

We chose this case study for its disastrous consequences that would have required the greatest possible number of
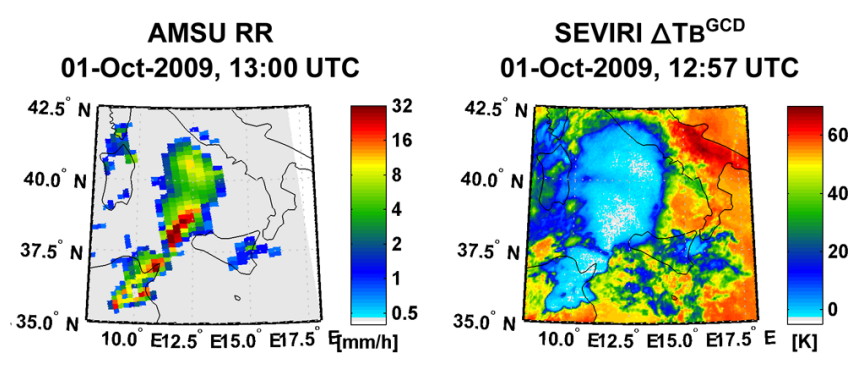

Fig. 1. AMSU/MHS rain-rate map from NOAA 18 with closest SEVIRI GCD map from SEVIRI.

monitoring tools. The storm was fully observed by the AMSU radiometers onboard NOAA and MetOp satellites only 4 times during the whole day, at 13:00, 13:05, 15:56 and 19:51 UTC, while it was continuously monitored every $15 \mathrm{~min}$ by the SEVIRI radiometer on the geostationary MSG satellite. To describe the PET algorithm for this case, precipitation estimates are derived by using MIT algorithm at 13:00 UTC and are propagated by using SEVIRI observations until 15:56 UTC, when the new AMSU/MHS overpass was available, neglecting AMSU/MHS overpass at 13:05 UTC because it was very close to the first. Figure 1 shows a precipitation retrieval obtained with MIT algorithm at 13:00 UTC with the closest GCD map at 12:57 UTC. In the following section we use only the MIT algorithm in conjunction with the PET algorithm because our purpose is to explain as PET works, while in Sect. 6 we use both the AMSU-based precipitation retrieval algorithms in order to show the different results produced by PET when different rain-rate data are used as input.

\section{The Precipitation Evolving Technique (PET)}

Figure 2 shows a general scheme of the PET algorithm. This algorithm is an iterative method that starts both with an AMSU/MHS overpass over the area for which rain field should be monitored and two consecutive SEVIRI observations. The first SEVIRI observation has to be contemporary to AMSU/MHS overpass, with a maximum tolerance in delay of $7.5 \mathrm{~min}$, that is half SEVIRI temporal sampling. By coupling the AMSU/MHS-based rain-rate map a $t=t_{0}$ with the GCD contemporary map $\Delta \mathrm{TB}_{t_{0}}^{\mathrm{GCD}}$, we obtain the first rain-rate map $\mathrm{RR}_{t_{0}}$ at IR scale that will be propagated by coupling the first GCD $\Delta \mathrm{TB}_{t_{0}}^{\mathrm{GCD}}$ map with the subsequent GCD map $\Delta \mathrm{TB}_{t_{0}+15^{\prime}}^{\mathrm{GCD}}$. MW-based rain rates are resampled to SEVIRI grid, by selecting the closest RR value for each IR grid point, in order to reduce the beam filling effects due to the coarse AMSU/MHS resolution and to take advantage of SEVIRI higher resolution. This downscaling simplifies the PET algorithm because it makes it possible to work always with the same spatial grid. Furthermore, the output of the 
algorithm that is rain field propagated forward in time and space, has a resolution greater than that used as input.

At the first iteration, by using $\mathrm{RR}_{t_{0}}, \Delta \mathrm{TB}_{t_{0}}^{\mathrm{GCD}}$ and $\Delta \mathrm{TB}_{t_{0}+15^{\prime}}^{\mathrm{GCD}}$ maps on the same grid, Morphing module and Calibration module produce two new maps $\mathrm{RR}_{t_{0}+15}^{\mathrm{mor}}$ and $\mathrm{RR}_{t_{0}+15}^{\mathrm{cal}}$ that, when combined, produce a final result $\mathrm{RR}_{t_{0}+15^{\prime}}$ on the SEVIRI grid. When a new SEVIRI observation is available a $t=t_{0}+30^{\prime}$, a new iteration is performed, starting with the $\mathrm{RR}_{t_{0}+15^{\prime}}$ output of the previous iteration coupled with $\Delta \mathrm{TB}_{t_{0}+15^{\prime}}^{\mathrm{GCD}}$ and $\Delta \mathrm{TB}_{t_{0}+30^{\prime}}^{\mathrm{GCD}}$, in order to produce a new rain-rate map $\mathrm{RR}_{t_{0}+30^{\prime}}$. Generally speaking, for each time step $t_{n}=t_{0}+n \Delta t$, with $\Delta t$ equal to SEVIRI temporal sampling, Morphing and Calibration modules use $\Delta \mathrm{TB}_{t_{0}+n \Delta t}^{\mathrm{GCD}}$ and $\Delta \mathrm{TB}_{t_{0}+(n+1) \Delta t}^{\mathrm{GCD}}$, to propagate $\mathrm{RR}_{t_{0}+n \Delta t}$ forward in time and space to obtain $\mathrm{RR}_{t_{0}+(n+1) \Delta t}$, until a new AMSU/MHS overpass is available and new $\mathrm{RR}_{t_{0}}$ estimates are produced.

\subsection{Morphing module}

The goal of this module is to drive precipitating cells of the latest rain-rate map by modifying their positions and shapes so as to derive a new rain-rate map, using a multi-scale and multi-threshold pattern recognition approach. The express purpose of the Morphing module is not to estimate a new rain field but to derive a morphed rain field starting from the previous one, so that, different MW-based rain-rate estimates can produce different results.

This module uses as input a rain-rate map $\mathrm{RR}_{t_{0}+n \Delta t}$ and two consecutive GCD maps $\Delta \mathrm{TB}_{t_{0}+n \Delta t}^{\mathrm{GCD}}$ and $\Delta \mathrm{TB}_{t_{0}+(n+1) \Delta t}^{\mathrm{GCD}}$ to produce as output a morphed rain-rate map $\mathrm{RR}_{t_{0}+(n+1) \Delta t}^{\text {mor }}$ and a morphed $\Delta \mathrm{TB}_{t_{0}+(n+1) \Delta t}^{\mathrm{GCD}, \mathrm{mor}}$ map. In detail, at the first step, the Morphing algorithm uses $\Delta \mathrm{TB}_{t_{0}+n \Delta t}^{\mathrm{GCD}}$ matrix to compute a flag matrix of $0 / 1 F_{t_{0}+n \Delta t}$, assigning for each pixel the value 1 if the corresponding pixel of $\Delta \mathrm{TB}_{t_{0}+n \Delta t}^{\mathrm{GCD}}$ is lower than a $P$-th percentile computed on a $(N \times N)$ pixel $^{2}$ box around the considered pixel, and 0 otherwise. Then this algorithm identifies all $M$ 8-connected regions $\left[\Delta \mathrm{TB}_{t_{0}+n \Delta t}^{\mathrm{GCD}}\right]^{m=1: M}$ of $\Delta \mathrm{TB}_{t_{0}+n \Delta t}^{\mathrm{GCD}}$ map by using a flag matrix $F_{t_{0}+n \Delta t}-$ where 8connected region means that for each pixel with flag matrix equal to 1 of the region, there is at least another pixel with flag matrix equal to 1 of the same region that is vertically, horizontally or diagonally contiguous to the considered one.

Thus, we can consider these 8-connected regions as representative of different precipitating regions, and the aim is to follow their evolutions in time and space. To this end, at the second step the Morphing module tries to identify the translational movements of the various 8-connected regions. This is accomplished by evaluating the sum of the difference modules of each 8-connected region $\left[\Delta \mathrm{TB}_{t_{0}+n \Delta t}^{\mathrm{GCD}}\right]^{m}$ with the corresponding region in $\Delta \mathrm{TB}_{t_{0}+(n+1) \Delta t}^{\mathrm{GCD}}$ matrix that is within $\mathrm{a} \sim 40 \mathrm{~km}$ radius, a requirement necessary to limit ambiguities in detecting cell movements (the $40 \mathrm{~km}$ value for $15 \mathrm{~min}$ corresponds to an upper limit of $160 \mathrm{~km} \mathrm{~h}^{-1}$ for the convective cells velocity). In a similar way, also the correlation index are evaluated between the smallest sub-matrices that include the 8-connected regions and the corresponding submatrices of $\Delta \mathrm{TB}_{t_{0}+(n+1) \Delta t}^{\mathrm{GCD}}$ matrix that are within a $\sim 40 \mathrm{~km}$ radius. Then, the translational movement having the minimum sum of the difference modules and the maximum correlation index is chosen and it is applied both to $\mathrm{RR}_{t_{0}+n \Delta t}$ and $\Delta \mathrm{TB}_{t_{0}+n \Delta t}^{\mathrm{GCD}}$.

In case of different translational movements generating the same values of sum of the difference modules and the correlation index, the smallest movement is preferred. When we have a cells overlap, the cell with the minimum value of $\Delta \mathrm{TB}_{t_{0}+n \Delta t}^{\mathrm{GCD}}$ maps and the maximum rain field value will be chosen. Once this computation is executed for all cells and the resulting translation movements are applied both to rain field and $\Delta \mathrm{TB}_{t_{0}+n \Delta t}^{\mathrm{GCD}}$ maps, the first computation of $\mathrm{RR}_{t_{0}+(n+1) \Delta t}^{\text {mor }}$ and $\Delta \mathrm{TB}_{t_{0}+(n+1) \Delta t}^{\mathrm{GCD} \text {,mor }}$ is done. At the end of this computation, the entire procedure is reiterated, varying flag matrix $F_{t_{0}+n \Delta t}$, by means of different $P$ percentiles and $N$ box sides, starting with the last computation of $\mathrm{RR}_{t_{0}+(n+1) \Delta t}^{\mathrm{mor}}$ and $\Delta \mathrm{TB}_{t_{0}+(n+1) \Delta t}^{\mathrm{GCD}, \mathrm{mor}}$ and updating their values.

$N$ and $P$ parameters have to vary by means of two nested loops, the first starts with a large value of $N$ and decreases it in some steps until a small box size is reached, whilst the second starts with a small $P$ value and increases it until a high percentile. By varying $N$ from high to small values, the Morphing algorithm tries to identify and move different cell scale sizes while varying $P$ from small to high values, it tries to identify and move the portions of the same cell differently. In doing so, for each iteration, translate regions gradually decrease in size and increase in number. In this way they produce, for each updating, a more and more accurate reconstruction of morphed rain-rate map $\mathrm{RR}_{t_{0}+(n+1) \Delta t}^{\mathrm{mor}}$ and morphed $\Delta \mathrm{TB}_{t_{0}+(n+1) \Delta t}^{\mathrm{GCD} \text { mor }}$ map.

Conceptually the best choice for nested loops is to start from $N$ equal to the maximum side of the examined area down to 2 pixels, and from $P$ equal to $1 \%$ up to $99 \%$, with a very small step for both $P$ and $N$ (i.e. 1 pixel and $1 \%$ ). To do it, computational cost may be too high for near realtime purposes. In the attempt to reach a good compromise between performance and computational cost, we chose to start with $N$ equal to the minimum side of the examined area and to decrease this value by $10 \%$ for each step, whilst for $P$ a good compromise is to start from $50 \%$ and to decrease this value by $5 \%$ for each step. Figure 3 shows examples of different $F_{t_{0}+n \Delta t}$ flag matrices for the adopted case study at 12:57 UTC and for some $N$ and $P$ values.

\subsection{Calibration module}

The goal of this module is to obtain a precipitation map a $t_{n}=t_{0}+(n+1) \Delta t$ by using the GCD-RR relationship dynamically computed at the previous time step $t_{n}=t_{0}+n \Delta t$. As 

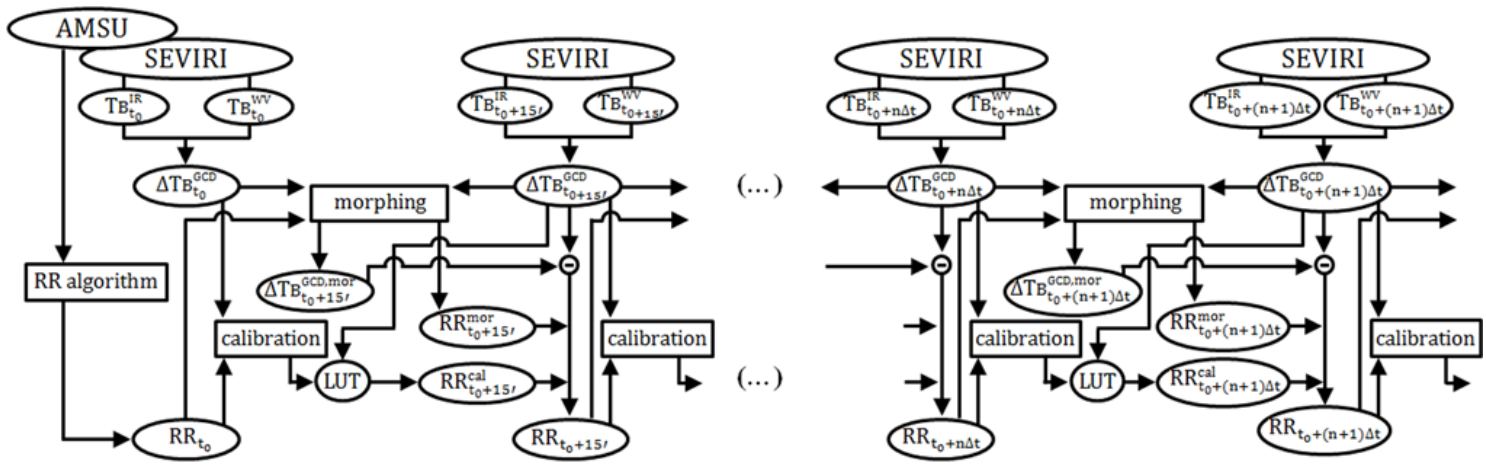

II $\mathbf{t}_{0}$

$\mathbf{t}_{0}+15$

Fig. 2. Scheme of the Precipitation Evolving Technique (PET).

previously explained for the Morphing module, Calibration module uses as input a rain-rate map $\mathrm{RR}_{t_{0}}+n \Delta t$ and two consecutive GCD maps $\Delta \mathrm{TB}_{t_{0}+n \Delta t}^{\mathrm{GCD}}$ and $\Delta \mathrm{TB}_{t_{0}+(n+1) \Delta t}^{\mathrm{GCD}}$ to produce as output a calibrated rain-rate map $\operatorname{RR}_{t_{0}+(n+1) \Delta t}^{\text {cal }}$. To this end, the Calibration module computes the $\mathrm{RR}_{t_{0}+n \Delta t}$ and $\Delta \mathrm{TB}_{t_{0}+n \Delta t}^{\mathrm{GCD}}$ histograms by using coincident rain-rate and GCD maps, then it uses a probabilistic histogram matching relationship (Calheiros and Zawadski, 1987) to calculate an RR- $\Delta \mathrm{TB}^{\mathrm{GCD}}$ lookup table (LUT). By applying this LUT to $\Delta \mathrm{TB}_{t_{0}+(n+1) \Delta t}^{\mathrm{GCD}}$ a new rain-rate map $\mathrm{RR}_{t_{0}+(n+1) \Delta t}^{\mathrm{cal}}$ is obtained. In some cases it may happen that $\Delta \mathrm{TB}_{t_{0}+(n+1) \Delta t}^{\mathrm{GCD}}$ contains values lower than those present in the calculated LUT, i.e. when there are convective events not completely covered by AMSU/MHS overpass but observed by SEVIRI radiometer, or when AMSU/MHS overpass observes convective cells before their complete development. For this reason, Calibration module uses also the last ten calculated LUTs jointly with a more general LUT obtained on the basis of 20 case studies selected in 2011. For each $\mathrm{RR}_{t_{0}+(n+1) \Delta t}^{\mathrm{cal}}$ computation, when the LUT does not cover all $\Delta \mathrm{TB}_{t_{0}+(n+1) \Delta t}^{\mathrm{GCD}}$ values, Calibration module searches these values in the other LUTs, starting from the latest up to the more general LUT. Obviously, in doing so, precipitation estimates are less accurate than those obtained by using only MW/IR simultaneous observations, but this approach allows recognizing precipitating areas also out of AMSU/MHS overpass or far from the mature stage.

\subsection{Precipitation results}

The PET algorithm final output is $\mathrm{RR}_{t_{0}+(n+1) \Delta t}$, obtained as a linear combination of $\mathrm{RR}_{t_{0}+(n+1) \Delta t}^{\text {mor }}$ and $\mathrm{RR}_{t_{0}+(n+1) \Delta t}^{\mathrm{cal}}$ computed pixel-by-pixel, on the same grid of IR data. In addition to the morphed rain field map $\mathrm{RR}_{t_{0}+(n+1) \Delta t}^{\mathrm{mor}}$, Morphing module produces also a morphed GCD map $\Delta \mathrm{TB}_{t_{0}+(n+1) \Delta t}^{\mathrm{GCD}, \text { mor }}$. The rain field $\mathrm{RR}_{t_{0}+(n+1) \Delta t}^{\mathrm{mor}}$ and GCD maps are obtained by identical sequences of translation movements calculated to get $\Delta \mathrm{TB}_{t_{0}+(n+1) \Delta t}^{\mathrm{GCD}}$ starting from $\Delta \mathrm{TB}_{t_{0}+n \Delta t}^{\mathrm{GCD}}$. Conceptually, if the differences of these two GCD maps were due only to the position of some cold areas, $\Delta \mathrm{TB}_{t_{0}+(n+1) \Delta t}^{\mathrm{GCD} \text {,mor }}$ would be a perfect reconstruction of $\Delta \mathrm{TB}_{t_{0}+(n+1) \Delta t}^{\mathrm{GCD}}$ and would be reasonable to consider $\mathrm{RR}_{t_{0}+(n+1) \Delta t}^{\text {mor }}$ as a perfect reconstruction of $\mathrm{RR}_{t_{0}+(n+1) \Delta t}$. In practice, for each SEVIRI observation, obviously $\triangle \mathrm{TB} \mathrm{GCD}^{\mathrm{G}}$ varies not only in the positions of cold areas, but also in shape and intensity, and consequently $\Delta \mathrm{TB}_{t_{0}+(n+1) \Delta t}^{\mathrm{GCD} \text {,mor }}$ and $\mathrm{RR}_{t_{0}+(n+1) \Delta t}^{\mathrm{mor}}$ are not perfect reconstructions of their corresponding fields. For this reason, the map of difference between $\Delta \mathrm{TB}_{t_{0}+(n+1) \Delta t}^{\mathrm{GCD} \text {,mor }}$ and $\Delta \mathrm{TB}_{t_{0}+(n+1) \Delta t}^{\mathrm{GCD}}$ can be considered as a measure of the Morphing module performance. In this map, the regions characterized by low values indicate a good Morphing module performances, whilst regions characterized by high values denote bad performance, that is a bad propagation of rain-rate maps. On the basis of these considerations, in order to combine $\mathrm{RR}_{t_{0}+(n+1) \Delta t}^{\mathrm{mor}}$ and $\mathrm{RR}_{t_{0}+(n+1) \Delta t}^{\mathrm{cal}}$ so as to obtain a final RR map PET algorithm uses

$\Delta \operatorname{mor}=\operatorname{abs}\left(\left.\Delta \mathrm{TB}_{t_{0}+(n+1) \Delta t}^{\mathrm{GCD}, \mathrm{mor}}\right|^{n}-\left.\Delta \mathrm{TB}_{t_{0}+(n+1) \Delta t}^{\mathrm{GCD}}\right|^{n}\right)$

where $\operatorname{abs}(\bullet)$ denotes absolute value and $\left.\bullet\right|^{n}$ indicates a matrix whose higher values than $n$ have been set equal to $n$. GCD maps are upper limited to $n$ value in order to evaluate the Morphing module performance only for cold regions that are associated to precipitating regions, neglecting large differences in warm regions.

Even if the GCD technique defines the convective regions with $\Delta \mathrm{TB}^{\mathrm{GCD}}>1 \mathrm{~K}$, we prefer to improve this value for security reasons in order to avoid skipping precipitating areas. For this paper $n=10$ is used, even if the differences in the final results are very limited, varying $n$ in the range [1/10]. Morphing module works well in the first applications of PET algorithm and its performance degrades over time as it moves away from AMSU/MHS overpass. This is an obvious consequence of how the Morphing module treats precipitating 


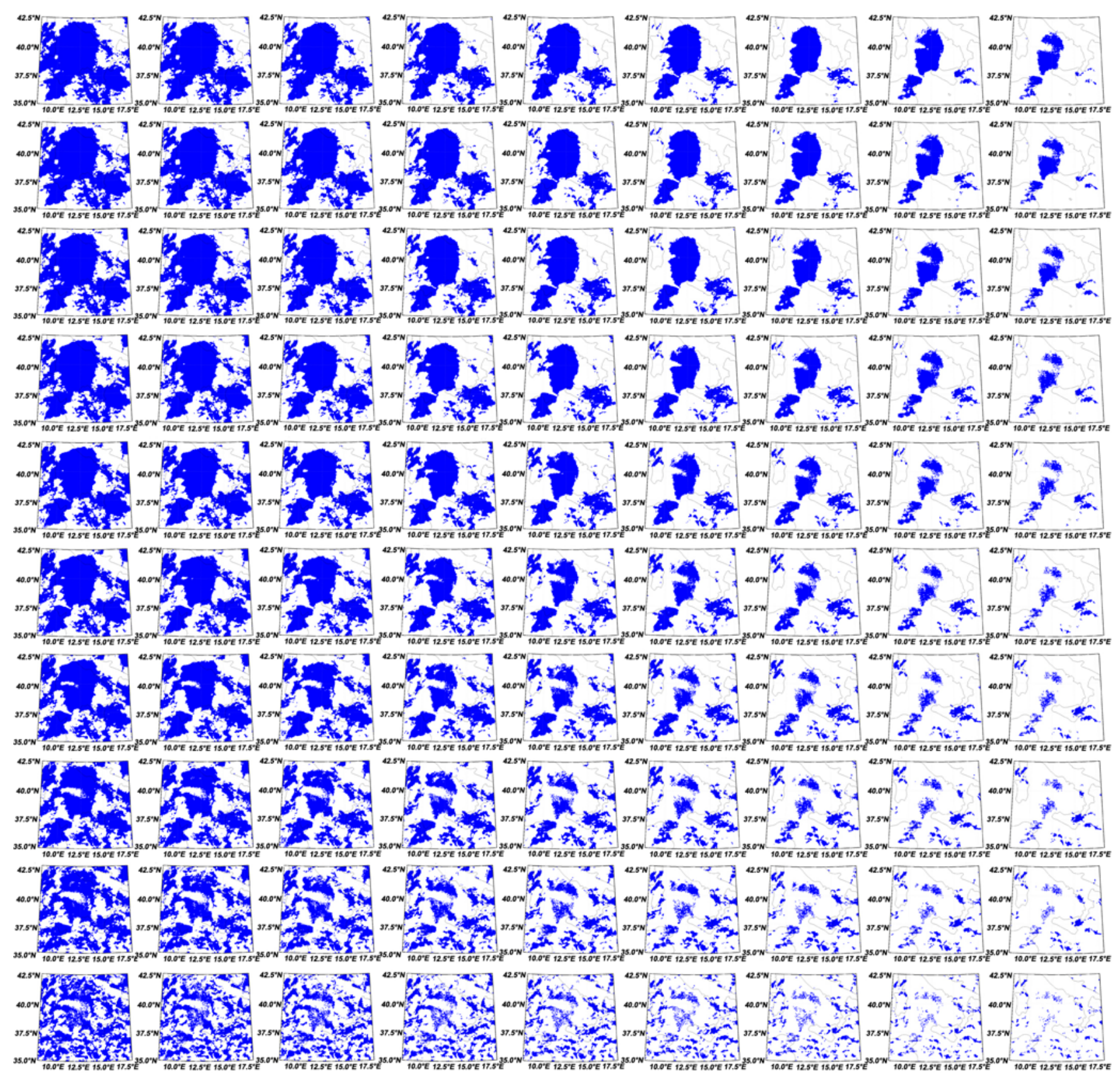

Fig. 3. Flag matrices computed on 1 October 2009 at 12:57 UTC, used for pattern recognition in Morphing module. From top to bottom $N$ box size decreases from $250 \mathrm{px}$ to $25 \mathrm{px}$ by step of $25 \mathrm{px}$, while from left to right $P$ percentile decreases from $50 \%$ to $10 \%$ by step of $5 \%$

cells, because it can recognize translations and changes in shape but not extinction, creation or large and quick variations of area. Moreover, Calibration module works less well than the other module in the first PET propagations, because it is based on statistic relationships but it is more stable over time. Because of this, in addition to $\Delta$ mor also a time factor $t$ mor is used in order to take into account the morphing mechanism progressive degradation and to combine the outputs of both modules. Good results are obtained by making heuristic choice of $t$ mor range from 0 to 1 with 12 regular steps, so that after $3 \mathrm{~h}$ only the Calibration output is used. In detail, to compute the final rain-rate map, PET algorithm uses $\Delta$ mor, scaling it between 0 and 1 by subtracting the minimum and dividing by the maximum value, and then it computes $C$ mor factor as $(1-\Delta$ mor $-t$ mor) limiting its value between 0 and 1 . The Final rain-rate map is: 

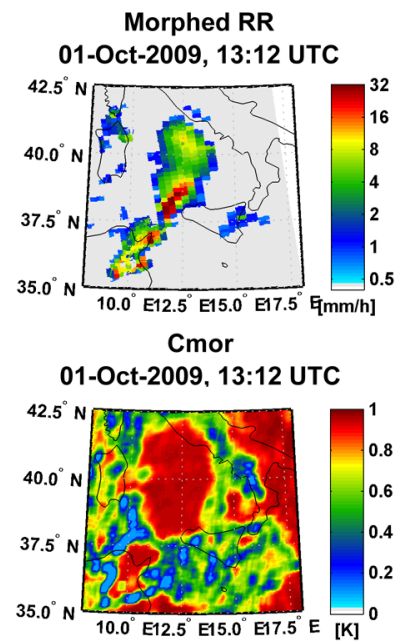

Calibrated RR 01-Oct-2009, 13:12 UTC

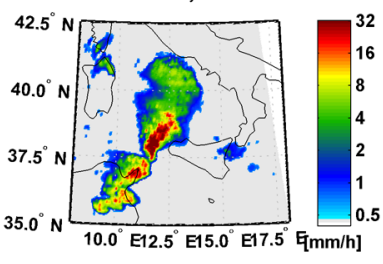

Final RR
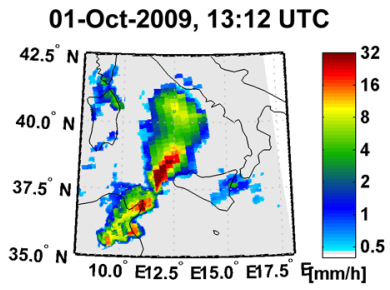

Fig. 4. Example of output of Morphing and Calibration modules, with coefficient matrix $C$ mor used to combine both outputs in final rain-rate map that is the result of first propagation of PET algorithm for event over on southern Italy, 1 October 2009.

$$
\begin{aligned}
\mathrm{RR}_{t_{0}+(n+1) \Delta t}= & C \text { mor } \cdot \mathrm{RR}_{t_{0}+(n+1) \Delta t}^{\mathrm{mor}}+(1-C \mathrm{mor}) \\
& \cdot \mathrm{RR}_{t_{0}+(n+1) \Delta t}^{\mathrm{cal}}
\end{aligned}
$$

In doing so, in the first PET propagations, precipitating regions with low differences between $\Delta \mathrm{TB}_{t_{0}+(n+1) \Delta t}^{\mathrm{GCD} \text {,mor }}$ and $\Delta \mathrm{TB}_{t_{0}+(n+1) \Delta t}^{\mathrm{GCD}}$ are propagated mainly by means of Morphing module, whilst regions with high differences between the previous values are propagated mainly by means of Calibration module. As time passes, $C$ mor goes to zero and only Calibration output becomes reliable. Figure 4 shows an example of Morphing and Calibration rainfall outputs, with $C$ mor matrix and the final rainfall output of PET algorithm for the adopted case study at 13:12 UTC that is the first propagation after the latest AMSU/MHS overpass.

\section{Application to the selected case study}

Figure 5 shows the precipitation evolution every $15 \mathrm{~min}$ on the SEVIRI grid, from 12:57 UTC to 15:42 UTC, as estimated by the PET algorithm starting from the rain field provided by MIT algorithm by using AMSU/MHS overpass at 13:00 UTC. In each panel $\Delta \mathrm{TB}^{\mathrm{GCD}}$ maps have also been included as references. The first panel shows an overall correlation between the pattern of precipitation, as provided by the AMSU/MHS-based algorithm, and the closest $\Delta \mathrm{TB}^{\mathrm{GCD}}$ map at 12:57 UTC. Pattern similarities are more evident where higher precipitations are estimated, such as over the Strait of Sicily. The correlation decreases with the decrease in precipitation intensity and the increase in $\triangle \mathrm{TB}^{\mathrm{GCD}}$ values, as over eastern Sicily or Sardinia. The good correlation between low

values of $\triangle \mathrm{TB}^{\mathrm{GCD}}$ map and heavy precipitating storms is a consequence of deep convection well recognized by the GCD approach. The low correlation between low precipitation intensity and medium-high $\triangle \mathrm{TB}^{\mathrm{GCD}}$ values is a consequence not only of shallow convection but also of the fact that MW remote-sensing is more efficient in observing high precipitation (Bennartz et al., 2006). For our purposes, this correlation is enough to hook precipitation to $\triangle \mathrm{TB}^{\mathrm{GCD}}$ and propagate the rain field by using SEVIRI data until a new precipitation estimation from one of the LEO satellites updates storm view.

In the following 5 panels of Fig. 5, from 13:12 UTC to 14:12 UTC, it is possible to observe that the coldest region of $\triangle \mathrm{TB}^{\mathrm{GCD}}$ tends to stretch and move toward the south and that the PET algorithm correctly follows this development, stretching and shifting the most intense precipitation cell to the Strait of Sicily, and decreasing the precipitation area over the Tyrrhenian Sea. It is worth considering that the PET algorithm restructures the shape of the low precipitation cell over Sardinia, remodulating it along the corresponding $\Delta \mathrm{TB}^{\mathrm{GCD}}$ cold region. In addition, the weak precipitation cell over Eastern Sicily at 13:00 UTC follows the $\triangle \mathrm{TB}^{\mathrm{GCD}}$ evolution falling rapidly. In the last 6 panels of Fig. 5, between 14:27 UTC and 15:42 UTC, one can observe a progressive movement eastward of the cold region of $\triangle \mathrm{TB}{ }^{\mathrm{GCD}}$, accompanied by the gradual decrease of the cold region over east Sicily and the Tyrrhenian Sea. This evolution of the $\triangle T^{G C D}$ map is well followed by the PET algorithm with the equivalent changes in the rain field, and the resulting formation of two high precipitation regions over western Sicily as well as the strong decrease in the low precipitation areas over the Tyrrhenian Sea and eastern Sicily.

\section{Evaluation of PET performances}

Generally speaking, the comparison with available radars or rain gauge measurements represents a good reference to understand the reliability of the satellite-based rain field estimates. This paper focuses on the evaluation of the PET algorithm in reproducing the AMSU/MHS-based rain-rate estimates when these instruments are not passing over the region. So that, to exclude the uncertainties related to the performance of the AMSU/MHS-based retrieval algorithm from the evaluation of PET results, it is more interesting to compare the PET performance versus the first available AMSU/MHS-based retrieved rain field successive to that used for initializing PET algorithm. For these reasons, both MIT and IMAA-CNR AMSU/MHS precipitation retrieval algorithms described in Sect. 2 are used, to further highlight the performances of PET, regardless of the MW-based retrieval rain rates used. The PET algorithm performances are evaluated in this section by means of the convective events listed in Table 2, both through a qualitative assessment, to verify the overall performance of the product, and continuous 

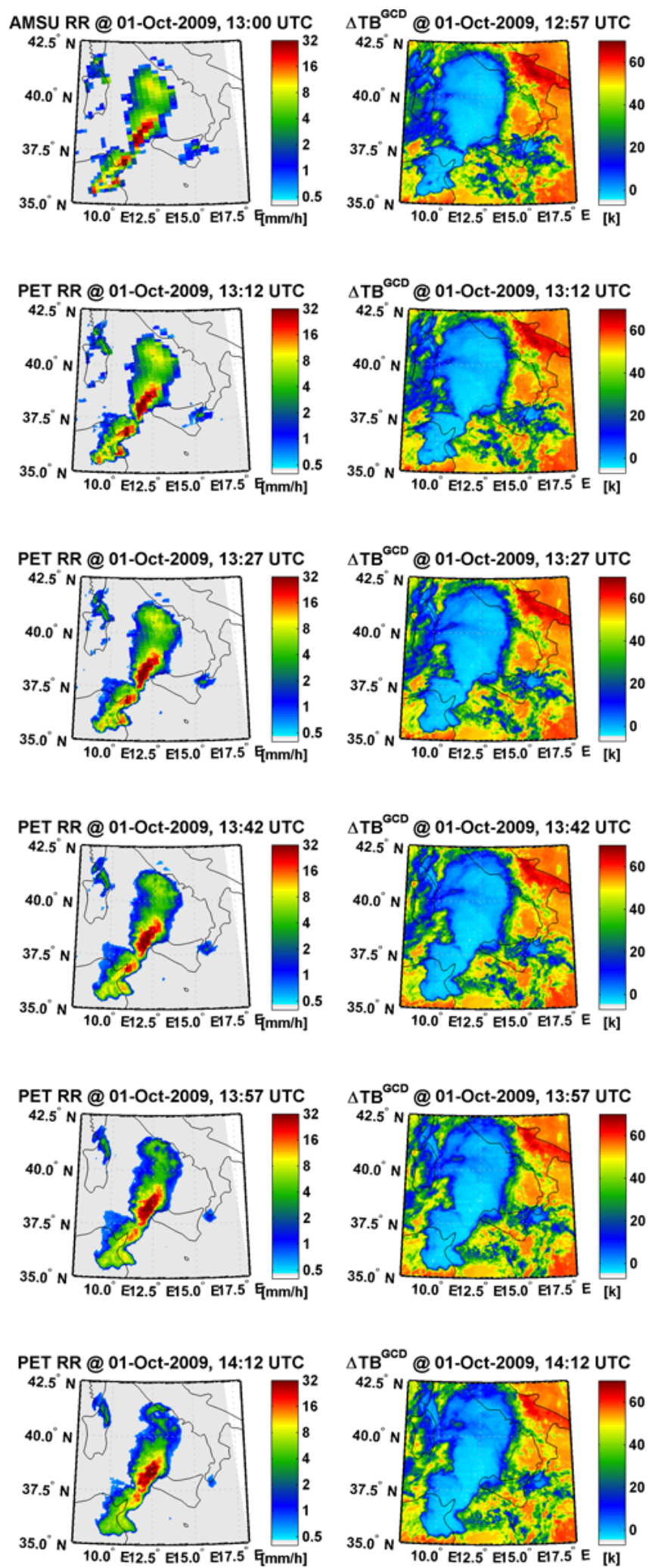
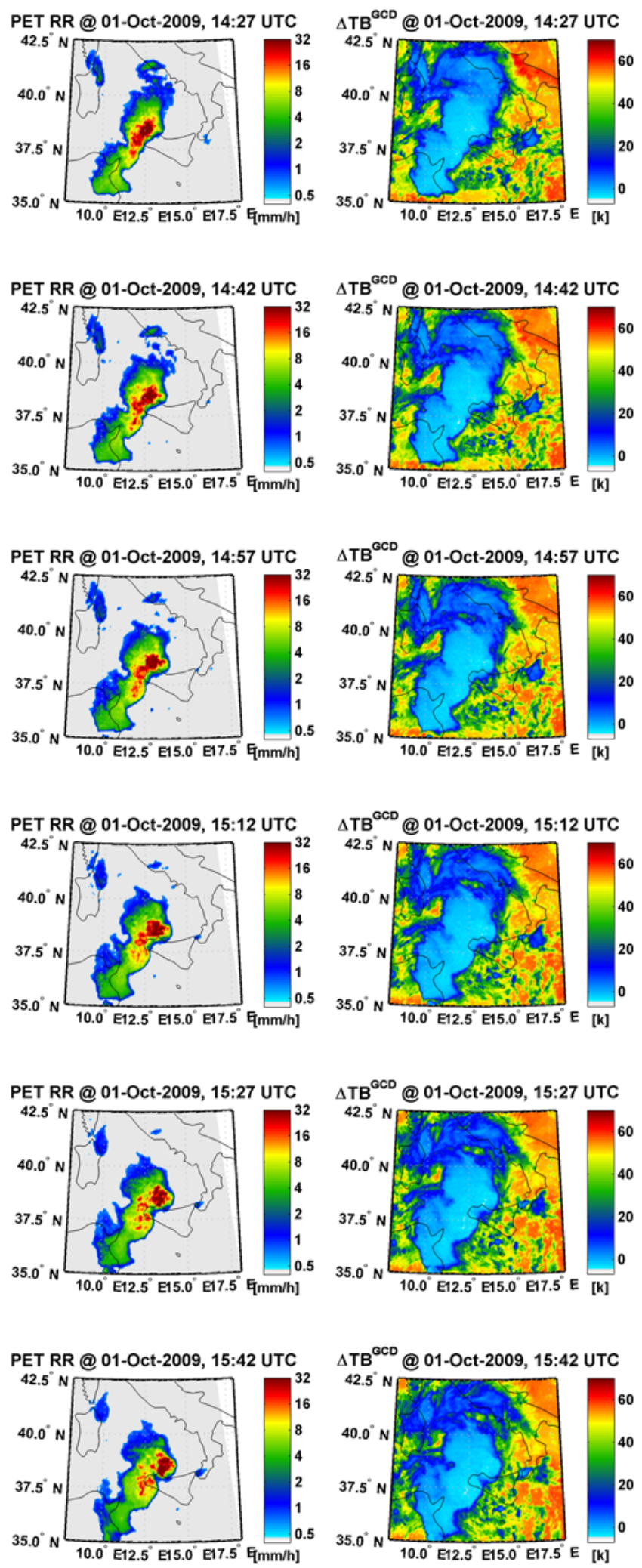

Fig. 5. Event over southern Italy, 1 October 2009. PET estimated rain-rate evolution from 13:12 UTC to 15:42 UTC, starting from the rain rates at 13:00 UTC provided by MIT algorithm applied to TB from AMSU/MHS onboard NOAA 18 satellite, and contemporary GCD maps from SEVIRI onboard MSG, used for PET algorithm 
Table 2. List of case studies selected for PET performance evaluations.

\begin{tabular}{llrl}
\hline $\begin{array}{l}\text { Case } \\
\text { study \# }\end{array}$ & $\begin{array}{l}\text { AMSU/MHS overpass } \\
\text { used for PET algorithm }\end{array}$ & $\begin{array}{r}\text { PET propagation } \\
\text { time }\end{array}$ & $\begin{array}{l}\text { AMSU/MHS overpass } \\
\text { used for comparison }\end{array}$ \\
\hline$(1)$ & $01-10-2009,13: 00$ UTC (NOAA-18) & $180^{\prime}$ & $01-10-2009,15: 54$ UTC (NOAA-15) \\
$(2)$ & $22-11-2010,09: 40$ UTC (MetOp-A) & $120^{\prime}$ & $22-11-2010,11: 46$ UTC (NOAA-19) \\
$(3)$ & $07-10-2011,09: 38$ UTC (MetOp-A) & $120^{\prime}$ & $07-10-2011,11: 39$ UTC (NOAA-19) \\
$(4)$ & $27-10-2011,20: 49$ UTC (MetOp-A) & $285^{\prime}$ & $28-10-2010,01: 32$ UTC (NOAA-19) \\
$(5)$ & $12-11-2011,08: 56$ UTC (MetOp-A) & $180^{\prime}$ & $12-11-2011,12: 00$ UTC (NOAA-19) \\
$(6)$ & $03-02-2012,20: 01$ UTC (MetOp-A) & $300^{\prime}$ & $04-02-2012,00: 55$ UTC (NOAA-19) \\
$(7)$ & $12-02-2012,01: 05$ UTC (NOAA-19) & $30^{\prime}$ & $12-02-2012,01: 35$ UTC (NOAA-18) \\
\hline
\end{tabular}
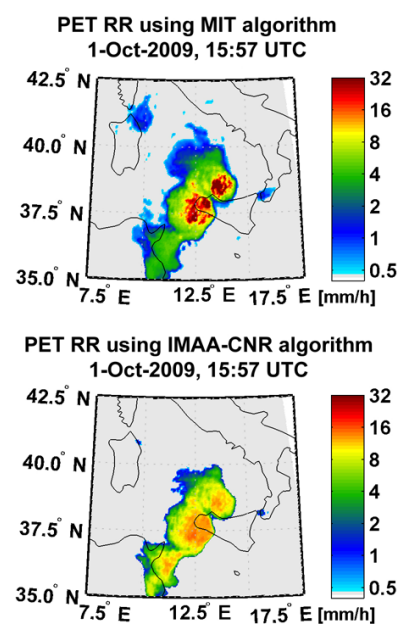
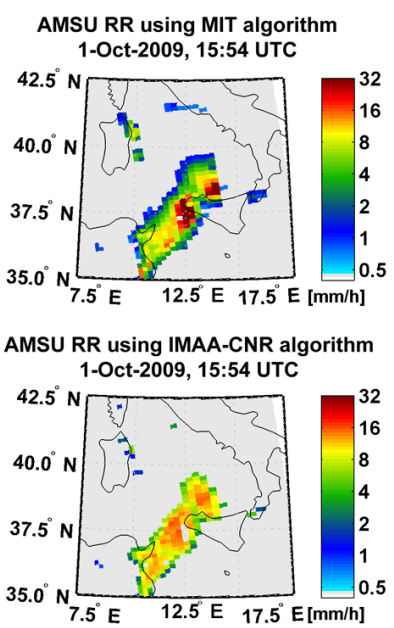

Fig. 6. Case study (1) - Comparison between PET rain-rate estimates obtained using both MIT and IMAA-CNR algorithm and closest corresponding AMSU/MHS-based rain-rate estimates. Gray area denotes first AMSU/MHS overpass.

and dichotomous statistical assessments, to verify the accuracy of rain-rate propagation. For the latter evaluation, PET output is spatially resampled to the AMSU/MHS overpass grid used for comparison.

For qualitative assessments, Fig. 6 shows the final results of PET rain field propagation compared with the closest AMSU/MHS-based rain field, by using both MIT and IMAA-CNR algorithms, for case study 1 . When comparing the results obtained by coupling PET and MIT algorithm with those obtained by coupling PET and IMAA-CNR algorithm, it is evident that PET is not an RR algorithm but rather a propagation method of starting rain field. The left panels of Fig. 6 show different distributions of rain-rate values and slightly different patterns. The PET results obtained with MIT algorithm have precipitations up to $\sim 30 \mathrm{~mm} \mathrm{~h}^{-1}$ and a large area of about $1 \mathrm{~mm} \mathrm{~h}^{-1}$, whilst those obtained with IMAA-CNR algorithm have a maximum rain rate at $\sim 15 \mathrm{~mm} \mathrm{~h}^{-1}$ and a smaller area of low precipitation. Comparing the right panels of Fig. 6 with the corresponding left panels, although the precipitation values are different

Table 3. Contingency table for the definition of statistics indexes POD, FAR, CSI, Bias and Accuracy.

\begin{tabular}{llll}
\hline & \multicolumn{2}{c}{ AMSU rain rate } \\
\cline { 3 - 4 } & & YES & NO \\
\hline \multirow{2}{*}{ PET rain-rate } & YES & Hits & False alarms \\
& NO & Misses & Correct negatives \\
\hline
\end{tabular}

pixel-by-pixel, the maps describe quite a similar precipitating pattern and similar values, showing an overall reliability of this technique.

The most interesting result is the correct identification of the two most intense cells, at $\sim 30 \mathrm{~mm} \mathrm{~h}^{-1}$ or $\sim 15 \mathrm{~mm} \mathrm{~h}^{-1}$, by using MIT and IMAA-CNR algorithms, respectively, and the tail of the main precipitation cell near the coast of Tunisia, even if over the Strait of Messina the values are a bit too intense for PET with MIT algorithm. The main problem that emerges in observing Fig. 6 is that the PET precipitation pattern is a bit too large, mostly at low precipitation areas, with a few pixels at low rain-rate values. Case studies number $2 / 7$, whose figures are omitted for simplicity, show similar behaviors, with an overall capability of this method in reproducing quite a similar precipitating pattern and similar values.

All case studies show, as expected by construction, that the Morphing module works well close to AMSU/MHS overpass, but its performance decays rapidly after a few minutes $(120 / 180)$. Conversely, Calibration module has a more regular performance with time, this works worse than Morphing module in the first $2-3 \mathrm{~h}$, but its performance does not decay as fast as that of the latter Qualitative analysis indicates an overall good agreement between the MW-based rain rates and contemporary PET rain rates.

For dichotomous assessment, in this paper we use the skill scores Probability Of Detection (POD), False Alarm Rate (FAR), Critical Success Index (CSI), Bias and Accuracy, which are categorical measures based on a rain contingency table (Table 3). POD is the number of hits divided by the total number of rain observations, and gives a simple measure of the rain events fraction that is correctly identified by PET algorithm. A perfect POD has a value of one and the 
Table 4. Dichotomous statistics results for all cases studies, obtained using both MIT and IMAA-CNR algorithms for PM precipitation retrieval.

\begin{tabular}{llrrrrrr}
\hline \multirow{2}{*}{ Statistics } & $\begin{array}{l}\mathrm{MW} \\
\text { Algorithm }\end{array}$ & $\begin{array}{c}\mathrm{RR}> \\
0 \mathrm{~mm} \mathrm{~h}\end{array}$ & $\begin{array}{c}\mathrm{RR}> \\
0.2 \mathrm{~mm} \mathrm{~h}^{-1}\end{array}$ & $\begin{array}{c}\mathrm{RR}> \\
1 \mathrm{~mm} \mathrm{~h}^{-1}\end{array}$ & $\begin{array}{c}\mathrm{RR}> \\
2 \mathrm{~mm} \mathrm{~h}^{-1}\end{array}$ & $\begin{array}{c}\mathrm{RR}> \\
5 \mathrm{~mm} \mathrm{~h}^{-1}\end{array}$ & $\begin{array}{c}\mathrm{RR}> \\
10 \mathrm{~mm} \mathrm{~h}^{-1}\end{array}$ \\
\hline \multirow{2}{*}{ POD } & MIT & 0.75 & 0.67 & 0.72 & 0.72 & 0.66 & 0.51 \\
& IMAA-CNR & 0.67 & 0.66 & 0.70 & 0.71 & 0.75 & 0.60 \\
\hline \multirow{2}{*}{ FAR } & MIT & 0.34 & 0.24 & 0.32 & 0.33 & 0.28 & 0.38 \\
& IMAA-CNR & 0.38 & 0.33 & 0.38 & 0.40 & 0.41 & 0.38 \\
\hline \multirow{2}{*}{ CSI } & MIT & 0.54 & 0.55 & 0.54 & 0.53 & 0.52 & 0.39 \\
& IMAA-CNR & 0.48 & 0.50 & 0.49 & 0.49 & 0.49 & 0.44 \\
\hline \multirow{2}{*}{ Bias } & MIT & 1.13 & 0.88 & 1.07 & 1.06 & 0.92 & 0.81 \\
& IMAA-CNR & 1.08 & 0.98 & 1.11 & 1.18 & 1.28 & 0.97 \\
\hline \multirow{2}{*}{ Accuracy } & MIT & 0.97 & 0.98 & 0.99 & 0.99 & 1.00 & 1.00 \\
& IMAA-CNR & 0.95 & 0.97 & 0.98 & 0.99 & 1.00 & 1.00 \\
\hline
\end{tabular}

worst possible POD has a value of zero. FAR is the number of false alarms divided by the total number of rain identified by PET algorithm, and gives a simple measure of the PET algorithm tendency to identify rain where none is observed. A perfect FAR is zero and the worst FAR is one. CSI is the number of hits divided by the total number of hits, misses and false alarms; it is a function of both FAR and POD, but unlike these, it takes both false alarms and missed events into account; therefore, it is a more balanced score. A perfect CSI is one and the worst CSI is zero. The Bias is the total number of rain identified by PET algorithm, divided by the total number of rain observations. The perfect PET rain identification with perfect Bias has a value of one, over estimation results in Bias greater than one, and under estimation results in Bias less than one. The Accuracy is the sum of hits and correct negatives identified by PET algorithm divided by the total number of rain observations. Perfect Accuracy is one and the worst Accuracy is zero.

Table 4 shows statistical scores obtained with all case studies for both MW-based rain-rate algorithms by using $0,0.2$, $1,2,5$ and $10 \mathrm{~mm} \mathrm{~h}^{-1}$ as rain/no-rain thresholds. The POD score shows that about $60-70 \%$ of area is detected rightly, while about $30 \%$ of the area detected as rainy is a false alarm (FAR). The POD is a little better for results obtained with MIT algorithm with respect to those obtained with IMAACNR algorithm, even if FAR is better for the last mentioned. This is probably a consequences of PM-RR retrieval algorithms rather than of PET algorithm, because IMAA-CNR algorithm appears a little more conservative with respect to MIT algorithm. Taking into account both POD and FAR, CSI indicates that the results obtained are roughly constant for different precipitation levels, except for precipitation with rain rates higher than $10 \mathrm{~mm} \mathrm{~h}^{-1}$, for which CSI is the worst, even if the total amount of pixels with high precipitation is low and then statistical scores are less reliable. Bias values are generally close to 1 , without a strong tendency to
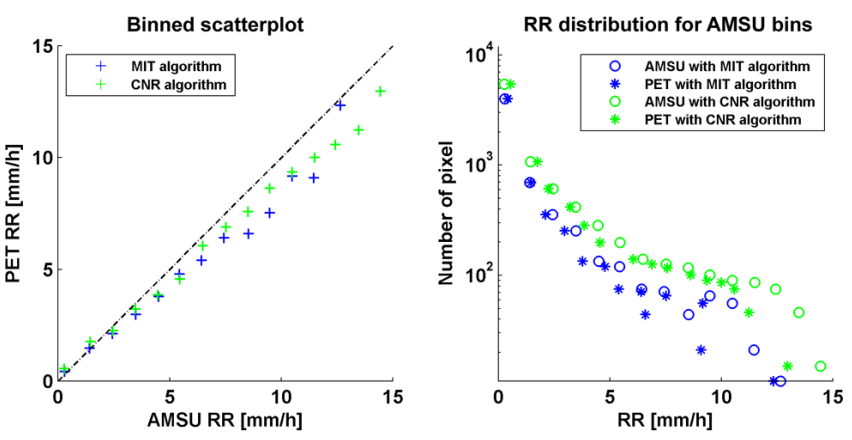

Fig. 7. All case studies - Binned scatter plot between average PET rain field estimates and $1 \mathrm{~mm} \mathrm{~h}^{-1}$ AMSU rain field estimates bins, using both MIT and IMAA-CNR algorithm with the corresponding RR distributions.

overestimate or underestimate the precipitation area. Accuracy values are close to 1 , to indicate a high probability to detect correctly both rain and no-rain events. The results obtained for each case study follow the trend described for the qualitative analysis, with better performances for cases with short propagation times. These results, conducted over a small set of case studies, show that PET algorithm is quite able to distinguish rainy from no-rainy areas.

For continuous assessment, Fig. 7 shows binned scatter plots of PET rain rates versus AMSU rain rates, for both PM precipitation algorithms, with relative rain-rate distributions. For this analysis the AMSU/MHS rain-rate data are binned in $1 \mathrm{~mm} \mathrm{~h}^{-1}$ rain-rate intervals and the PET rain rates in these bins are averaged for all the case studies. The analysis is performed in order to place equal emphasis on the whole rainrate range as well as to minimise match-up errors between PET rain rates and AMSU/MHS rain rates. This scatter plot is quite regular, it is closer to the bisector and shows a reasonably good correlation between AMSU rain rates and PET 
Table 5. Continuous statistics results.

\begin{tabular}{llccc}
\hline $\begin{array}{l}\text { Case } \\
\text { study \# }\end{array}$ & MW & $R^{2}$ & RMSE & MAE \\
\hline \multirow{2}{*}{$(1)$} & MIT & 0.90 & 0.73 & 0.56 \\
& IMAA-CNR & 0.94 & 0.68 & 0.59 \\
$(2)$ & MIT & 0.96 & 0.65 & 0.49 \\
& IMAA-CNR & 0.95 & 0.76 & 0.58 \\
$(3)$ & MIT & 0.99 & 0.13 & 0.11 \\
& IMAA-CNR & 0.98 & 0.54 & 0.42 \\
$(4)$ & MIT & 0.88 & 0.50 & 0.47 \\
& IMAA-CNR & 0.95 & 0.28 & 0.24 \\
$(5)$ & MIT & 0.94 & 0.41 & 0.34 \\
& IMAA-CNR & 0.87 & 0.38 & 0.33 \\
$(6)$ & MIT & 0.94 & 0.36 & 0.26 \\
& IMAA-CNR & 0.96 & 0.55 & 0.46 \\
(7) & MIT & 0.98 & 0.21 & 0.19 \\
& IMAA-CNR & 0.97 & 0.48 & 0.37 \\
All cases & MIT & 0.97 & 0.54 & 0.35 \\
& IMAA-CNR & 0.99 & 0.25 & 0.21 \\
\hline \multirow{2}{*}{ (5) }
\end{tabular}

rain rates, even if there is a small overestimate of low precipitation bins and a small underestimate of high precipitation bins. This result is a consequence of this method that uses an average of PET rain rates for each AMSU rain-rate bin, rather than a true overestimate/underestimate, as shown by the rain-rate distribution in the right panel. Indeed, rain-rate distribution shows that PET rain rates has approximately the same AMSU/MHS rain-rate distribution, for both MIT and IMAA-CNR algorithms, even if these have a slightly different distribution between them. Final PET rain-rate distribution depends heavily on the PM rain-rate retrieval algorithm used, thus demonstrating that PET is a rain-rate propagation method rather than an algorithm for rain-rate estimates.

Table 5 shows continuous statistics results for binned scatter plot obtained by neglecting bins with less than 10 pixels. The square of the correlation coefficient $\left(R^{2}\right)$, the root-mean squared error (RMSE), and the mean absolute error (MAE), computed for a linear fit for the individual case studies and for all the case studies together, show that the PET algorithm produces quite good results in rain-rate propagation. In particular when all the case studies are considered, results are good with $R^{2}$ very close to 1 and RMSE and MAE less than 1. Obviously, PET performs better in some cases studies than in others, depending on PET propagation time and in a way that might depend on the type of event.

To sum up, PET algorithm shows a good ability to propagate rain rates depending on the propagation time primarily, as well as on the nature of the storm under consideration. While its performance is acceptable for $2-3 \mathrm{~h}$ propagation time, it may become unsatisfactory after $4-5 \mathrm{~h}$.

\section{Summary and conclusions}

PET is a combined MW-IR method that exploits the ability of PM from LEO satellites to estimate convective precipitation with high temporal sampling of IR observations from GEO satellites. By using a starting rain field obtained from AMSU/MHS, PET produces a rain-rate map for each SEVIRI observation, so that this method fills the temporal gap existing between two consecutive AMSU/MHS overpasses with several rain-rate maps. Due to the possibility of being used every 15 min during daytime and nighttime, PET algorithm can become useful for nowcasting experts and flood alarm managers.

PET algorithm, initialized by two different AMSU/MHSbased precipitation retrieval algorithms produces different results, thus demonstrating its ability to propagate starting rain field rather than estimate it. In addition, the structure in two separate modules of Morphing and Calibration allows PET to propagate the rain field also out of AMSU/MHS overpass. These features could be used in future works to propagate different kinds of rain field, such as those obtained from ground-based network of radar or rain gauges, for real-time rain rates monitoring also for the area which is not covered by them.

No radar or rain gauges measurements have been used for PET validation, but the AMSU/MHS-based rain rates were used as the "truth", in order to separate the effects strictly related to PET algorithm from those due to the limited accuracy of the MW retrievals. Seven case studies have been shown to highlight the PET performances, demonstrating an overall agreement between PET rain field and the "truth" rain field, at least for a propagation time of $2-3 \mathrm{~h}$, while for longer times PET results are unsatisfactory although a more in-depth analysis would be necessary. A qualitative assessment has shown a good capability of PET to monitor the pattern and the intensity evolution of rain-rate maps, even if in some cases the first seemed a bit too large. Dichotomous assessment performed for six rain/no-rain thresholds has shown a CSI score of about $50 \%$, with a good POD score of about $70 \%$ but with a bit too high a FAR score of about $35 \%$, whilst Bias and Accuracy are close to 1 . Continuous assessment performed for binned rain-rate intervals has shown the scatter plots of PET rain rates versus AMSU-based rain rates quite close to the bisector, with $R^{2}$ of about 0.98 , RMSE of about 0.40 and MAE 0.28 .

Even if we plan to perform an extensive validation of PET results by means of ground-based and space-borne radars, such as the TRMM PR and rain gauge networks, the PET capability of hooking the precipitation cells evolution has been shown in this work, and it is an evident characteristic which is worth exploiting in satellite-based monitoring. 
Acknowledgements. This research activity has been supported by the Italian Civil Protection Agency.

Edited by: G. Boni

Reviewed by: two anonymous referees

\section{References}

Adler, R. F., Keehn, P. R., and Hakkarinen, I. M.: Estimation of monthly rainfall over Japan and surrounding waters from a combination of low-orbit microwave and geosynchronous IR data, J. Appl. Meteor., 32, 335-356, 1993.

Adler, R. F., Huffman, G. J., and Keehn, P. R.: Global tropical rain estimates from microwave-adjusted geosynchronous IR data, Remote Sens. Rev., 11, 125-152, 1994.

Aminou, D. M. A., Jacquet, B., and Pasternak, F.: Characteristics of the Meteosat Second Generation (MSG) Radiometer/Imager: SEVIRI, Proceeding of SPIE, Europto series, 3221, 19-31, 1997.

Bellerby, T. M., Todd, T., Kniveton, D., and Kidd, C.: Rainfall estimation from a combination of TRMM precipitation radar and GOES multispecral satellite imagery through the use of an artificial neural network, J. Appl. Meteor., 39, 2115-2128, 2000.

Bennartz, R., Thoss, A., Dybbroe, A., and Michelson, D. B.: Precipitation analysis using the Advanced Microwave Sounding Unit in support of Nowcasting applications, Meteorol. Appl., 9, 177$189,2006$.

Bizzarri, B., Gasiewski, A. J., and Staelin, D. H.: Observing rain by millimetre-submillimetre wave sounding from geostationary orbit, in: Measuring Precipitation from Space - EURAINSAT and the Future, edited by: Levizzani, V., Bauer, P., and Turk, F. J., Springer, 675-692, 2007.

Calheiros, R. V. and Zawadzki, I.: Reflectivity rain-rate relationship for radar hydrology and Brazil, J. Clim. Appl. Meteor., 26, 118132, 1987.

Casella, D., Dietrich, S., Di Paola, F., Formenton, M., Mugnai, A., Porcù, F., and Sanò, P.: PM-GCD - a combined IR-MW satellite technique for frequent retrieval of heavy precipitation, Nat. Hazards Earth Syst. Sci., 12, 231-240, doi:10.5194/nhess-12-2312012, 2012.

Di Paola, F. and Dietrich, S.: Resolution enhancement for microwave-based atmospheric sounding from geostationary orbits, Radio Sci., 43, RS6001, doi:10.1029/2007RS003789, 2008.

Di Tomaso, E., Romano, F., and Cuomo, V.: Rainfall estimation from satellite passive microwave observations in the range $89 \mathrm{GHz}$ to $190 \mathrm{GHz}$, J. Geophys. Res., 114, D18203, doi:10.1029/2009JD011746, 2009.

Dietrich, S., Casella, D., Di Paola, F., Formenton, M., Mugnai, A., and Sanò, P.: Lightning-based propagation of convective rain fields, Nat. Hazards Earth Syst. Sci., 11, 1571-1581, doi:10.5194/nhess-11-1571-2011, 2011.

Ebert, E. E., Manton, M. J., Arkin, P. A., Allam, R. J., Holpin, G. E., and Gruber, A. J.: results from the GPCP Algorithm Intercomparison Programme, B. Am. Meteor. Soc., 77, 2875-2887, 1996.

Huffman, G. J., Adler, R. F., Morrisey, M. M., Bolvin, D. T., Curtin, S., Joyce, R., McGavock, B., and Susskind, J.: Global precipitation at one-degree daily resolution from multisatellite observations, J. Hydrometeor., 2, 36-50, 2001.
Huffman, G. J., Adler, R. F., Curtis, S., Bolvin, D. T., and Nelkin, E. J.: Global rainfall analyses at monthly and 3-h time scales, in: Measuring Precipitation from Space - EURAINSAT and the Future, edited by: Levizzani, V., Bauer, P., and Turk, F. J., Springer, 291-305, 2007.

Joyce, R. J., Janowiak, J. E., Arkin, P. A., and Xie, P.: CMORPH: A method that produces global precipitation estimates from passive microwave and infrared data at high spatial and temporal resolution, J. Hydrometeor., 5, 487-503, 2004.

Joyce, R. J., Janowiak, J. E., Xie, P., and Arkin, P. A.: CPC MORPHING technique (CMORPH), in: Measuring Precipitation from Space - EURAINSAT and the Future, edited by: Levizzani, V., Bauer, P., and Turk, F. J., Springer, 307-317, 2007.

Kidd, C. K., Kniveton, D. R., Todd, M. C., and Bellerby, T. J.: Satellite rainfall estimation using combined passive microwave and infrared algorithms, J. Hydrometeor., 4, 1088-1104, 2003.

Kidd, C. K., Tapiador, F. J., Sanderson, V., and Kniveton, D.: The University of Birmingham global rainfall algorithms, in: Measuring Precipitation from Space - EURAINSAT and the Future, edited by: Levizzani, V., Bauer, P., and Turk, F. J., Springer, 255267, 2007.

Kleespies, T. J. and Watts, P.: Comparison of simulated radiances, jacobians and linear error analysis for the Microwave Humidity Sounder and the Advanced Microwave Sounding Unit-B, Q. J. R. Meteorol. Soc., 132, 3001-3010, 2007.

Kramer, H. J.: Observation of the Earth and Its Environment, Springer-Verlag, 1510 pp., 2002.

Kubota, T., Shige, S., Hashizume, H., Aonashi, K., Takahashi, N., Seto, S., Hirose, M., Takayabu, Y. N., Nakagawa, K., Iwanami, K., Ushio, T., Kachi, M., and Okamoto, K.: Global precipitation map using satelliteborne microwave radiometers by the GSMaP project: production and validation, IEEE Trans. Geosci. Remote Sens., 45, 2259-2275, 2007.

Kuligowski, R. J.: A self-calibrating real-time GOES rainfall algorithm for short-term rainfall estimates, J. Hydrometeor., 3, 112 130, 2002.

Kummerov, C., Hong, Y., Holson,W. S., Yang, S., Adler, R. F., McCollum, J., Ferraro, R., Petty, G., Schin, D.-B., and Wilheit, T. T.: The evolution of the Goddard profiling algorithm (GPROF) forrainfall estimation from passive microwave sensors, J. J. Appl. Meteor., 40, 1801-1820, 2001.

Martin, D. W., Kohrs, R. A., Mosher, F. R., Medaglia, C. M., and Adamo, C.: Over-Ocean Validation of the Global Convective Diagnostic, J. Appl. Meteor. Climatol., 47, 525-543, 2008.

Miller, S. W., Arkin, P. A., and Joyce, R.: A combined microwave/infrared rain rate algorithm, Int. J. Remote. Sens., 22, 3285-3307, 2001.

Mosher, F. R.: A satellite diagnostic of global convention, Preprints, 11th Conf on Satellite Meteorology, Madison, WI, Am. Meteor. Soc., 416-419, 2001.

Mosher, F. R.: Detection of deep convection around the globe, Preprints, 10th Conf. on Aviation, Range and aerospace Meteorology, Portland, OR, Am. Meteor. Soc., 289-292, 2002.

Muller, J.: MSG Level 1.5 Image Data Description, EUMETSAT, technical document number EUM/MSG/ICD/105, 2007.

Rosenkranz, P. W.: Radiative transfer solution using initial values in a scattering and absorbing atmosphere with surface reflection, IEEE Trans. Geosci. Remote Sens., 40, 1889-1892, 2002. 
Smith, E. A., Lamm, J. E., Adler, R. F., Alishouse, J., and Aonashi, K.: Results of the WetNet PIP-2 project, J. Atmos. Sci., 55, 14831536, 1998.

Sorooshian, S., Hsu, K.-L., Gao, X., Gupta, H. V., Imam, B., and Braithwaite, D.: Evaluation of PERSIANN system satellitebased estimates of tropical rainfall, B. Am. Meteor. Soc., 81, 20352046, 2000.

Surussavadee, C. and Staelin, D. H.: Global millimeter-wave precipitation retrievals trained with a cloud-resolving numerical weather prediction model, Part I: Retrieval design, IEEE Trans. Geosci. Remote Sens., 46, 99-108, 2008a.

Surussavadee, C. and Staelin, D. H.: Global millimeter-wave precipitation retrievals trained with a cloud-resolving numerical weather prediction model, Part II: Performance evaluation, IEEE Trans. Geosci. Remote Sens., 46, 109-118, 2008 b.

Torricella, F., Levizzani, V., and Turk, F. J.: Application of a blended MW-IR rainfall algorithm to the Mediterranean, in: Measuring Precipitation from Space - EURAINSAT and the Future, edited by: Levizzani, V., Bauer, P., and Turk, F. J., Springer, 497-507, 2007.
Turk, F. J. and Mehta, A. V.: Towards improvements in shorttime scale satellite-derived precipitation estimates using blended satellite techniques, in: Measuring Precipitation from Space EURAINSAT and the Future, edited by: Levizzani, V., Bauer, P., and Turk, F. J., Springer, 281-290, 2007.

Turk, F. J. and Miller, S. D.: Toward improving estimates of remotely-sensed precipitation with MODIS/ AMSR-E blended data techniques, IEEE T. Geosci. Remote Sens., 43, 1059-1069, 2005.

Turk, F. J., Ebert, E. E., Sohn, B.-J., Oh, H.-J., Levizzani, V., Smith, E. A., and Ferraro, R. R.: Validation of an operational global precipitation analysis at short time scales, Proc. 1st IPWG Workshop, Madrid, 23-27 Sept., 225-248, 2002.

Xu, L., Gao, X., Sorooshian, S., Arkin, P. A., and Imam, B.: A microwave infrared threshold technique to improve the GOES precipitation index, J. Appl. Meteor., 38, 569-579, 1999. 